Review

\title{
Relevance of Platelet-independent Effects of Aspirin to Its Salutary Effect in Atherosclerosis-related Events
}

\author{
Qaisar Khan, and Jawahar L. Mehta \\ Division of Cardiovascular Medicine, University of Arkansas for Medical Sciences College of Medicine and the \\ Central Arkansas Veterans Healthcare System, AR, USA.
}

\begin{abstract}
There is a close inter-relationship between oxidative stress, coagulation, inflammation, and smooth muscle cell growth as key components of atherosclerosis (Fig. 1). As an analgesic and anti-pyretic, aspirin has been in use for over a century. It acetylates the COX enzyme, irreversibly inhibiting the formation of prostaglandin. Its action on platelet $\mathrm{TxA}_{2}$ has highlighted its role as an anti-thrombotic agent in cardiovascular patients. Over the last two decades, unique anti-inflammatory properties of aspirin not shared by other non-steroidals have been discovered. Aspirin biotransforms into salicylate, which has diverse but potent anti-inflammatory properties. As we strive to better understand the concepts of atherogenesis, chronic inflammation, oxidative stress, and endothelial activation, these novel effects of aspirin provide new insights as to how this wonder drug works. These effects of aspirin alter many, if not all, components of the atherogenesis cascade shown in Fig. 1. J Atheroscler Thromb, 2005; 12: 185-190.
\end{abstract}

Key words: Adesion molecules, Inflammation, oxidative stress, smooth muscle cell growth

\section{Introduction}

In this era of ever-increasing poly-pharmaceutical approaches to cardiovascular diseases, aspirin has come to be the leading drug in the reduction of cardiovascular mortality, followed by beta-blockers, statins and ACEinhibitors. Aspirin's widespread use as a primary and secondary preventive agent for cardiovascular disease has been well established among physicians. It reduces the evolution of vascular events by $20-25 \%$, and commands a class I indication in the treatment of acute coronary syndrome by the American Heart Association/American College of Cardiology Task Force $(1,2)$.

The medicinal benefits of aspirin have been known to mankind for over a century, long before it's mechanism of action was understood (3). Salicin was in therapeutic use in the form of white willow bark extract for almost

$\overline{\text { Address for correspondence: J. L. Mehta, University of Arkansas }}$ for Medical Sciences, Department of Internal Medicine, 4301 West Markham, Slot 532, Little Rock, AR 72205, USA.

E-mail: MehtaJL@uams.edu

Received April 8, 2005

Accepted for publication April 19, 2005
2000 years. Acetylsalicylic acid was first synthesized in the 1850 s for use in treating rheumatism as an anti-inflammatory, analgesic and anti-pyretic agent. It was in 1971 that Sir John Vane demonstrated that the inhibition of prostaglandin synthesis was the mechanism of action of aspirin. It is now well accepted that the beneficial effects of aspirin in the secondary prevention of myocardial infarction are primarily mediated by an irreversible inhibition of the cyclooxygenase-1 (COX-1) enzyme, resulting in the inhibition of thromboxane $\mathrm{A}_{2}\left(\mathrm{TXA}_{2}\right)$ formation during the life span of platelets which are unable to regenerate COX-1.

Aspirin has found a unique place in the treatment of atherosclerosis and its manifestations which result from an interaction between platelet activation, endothelial inflammation/activation, and oxidative stress (Fig. 2). The usual risk factors for coronary atherosclerosis, which include diabetes mellitus, hypertension, dyslipidemia and smoking, are associated with intense oxidative stress to intense inflammation. It is critical to understand the process of atherogenesis and events that lead to plaque rupture to understand the benefits of aspirin therapy. We 


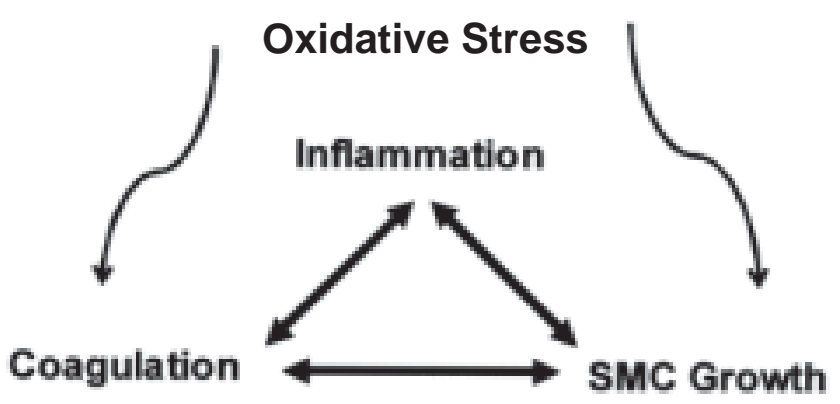

Fig. 1. Inter-relationship between oxidative stress, inflammation, coagulation and smooth muscle cell growth.

review here some of the work that shows aspirin to be a potent vasoprotective agent through its well known platelet-dependent and the emerging platelet-independent mechanisms.

\section{Platelet-dependent Effects of Aspirin}

The direct anti-thrombotic effect of aspirin is believed to be mediated by inhibition of the COX-1 enzyme in platelets (4). This effect is mediated by the irreversible acetylation of COX-1 at a serine residue near the catalytic site, permanently altering the site for arachidonic acid resulting in a decreased production of $\mathrm{TxA}_{2}$, a potent vasoconstrictor and platelet pro-aggregant (5). This irreversible inhibition of COX-1 provides a sufficient explanation for the selective inhibition of COX by low-dose aspirin in platelets that, in contrast to vascular endothelial cells, cannot synthesize COX de novo.

This anti-platelet effect presumably translates into significant clinical benefits in patients with unstable angina, acute myocardial infarction, coronary artery bypass graft and percutaneous coronary interventions. In all these conditions, there is evidence for enhanced platelet aggregation. Although most of the benefits of aspirin in vascular disease have been attributed to inhibition of platelet $\mathrm{TxA}_{2}$ synthesis, it should be noted that direct $\mathrm{TxA}_{2}$ receptor antagonists have not been found to be of any clinical benefit (6). Further, other agents that inhibit the COX enzyme such as indomethacin or ibuprofen do not exert the same cardioprotective effects as aspirin (6). These observations raise concern regarding the major mechanisms by which aspirin exerts its salutary effect.

\section{Platelet-independent Effects of Aspirin}

\section{Effect on oxidative stress}

There is compelling evidence that an excess of oxidative stress beyond the body's ability to cope contributes significantly to endothelial damage during atherogenesis. Oxidant burden seems to correlate with cardiovascular risk factors $(7,8)$. Oxidative stress is a result of the release of reactive oxygen species (ROS) and other nitrosylated products. ROS, in particular, mediates atherogenic signals in vascular cells. Growth factors such as angiotensin II also stimulate production of ROS (8) which activate several intracellular responses, including the activation of NF- $\kappa B$ with the subsequent upregulation of adhesion molecules $(9,10)$. A number of studies show that aspirin, but not indomethacin, may exert an inhibitory effect on the generation of ROS and associated intercellular signaling pathways $(11,12)$.

The atherosclerotic arteries also exhibit the deposition of oxidatively modified low density lipoproteins (ox-LDL) (13) which along with shear stress, endothelin and angiotensin II, upregulate the activation of ox-LDL-specific receptors, such as LOX-1, leading to endothelial activation, dysfunction, apoptosis, and MMP-1 synthesis as well as platelet activation (14).

We have observed that aspirin, in a dose- and timedependent fashion, reduced ox-LDL-mediated LOX-1 expression, MMP-1 expression and activity, p38MAPK activation and superoxide anion generation in human coronary artery endothelial cells (15). Interestingly, we have observed that the treatment of human coronary artery endothelial cells with salicylate, but not indomethacin, resulted in effects similar to those of aspirin (15).

In earlier studies, Chen et al. (16) demonstrated in our laboratory that ox-LDL caused a concentration-dependent increase in thrombin-induced platelet aggregation and decreased activity. These effects were reversed by the pretreatment of platelets with L-arginine, the precursor (Fig. 3). O'Kane et al. (17) showed that basal NOS activity in human platelets was increased significantly by intravenous aspirin. In the same study, incubation with aspirin, but not with indomethacin or ibuprofen, increased NOS activity in platelets, suggesting that aspirin acts through a mechanism independent of COX inhibition. It is well known that oxidative stress causes the breakdown of NO.

Grosser and Schröder (18) showed that preincubation with aspirin, but not salicylate or indomethacin, protected endothelial cells from hydrogen peroxide-mediated toxicity and increased their viability in a concentration-dependent fashion. This effect was abrogated in the presence of a NO scavenger and L-arginine analogs. They also showed that aspirin enhanced activity and intracellular cyclic GMP accumulation in endothelial cells (18). Similar protection of endothelial cells from oxidative stress by vitamin E had been shown earlier (19). Aspirin has also been shown to prevent hydrogen peroxide-induced caspase-3, caspase- 9 and NF- $\mathrm{KB}$ activation in a dose-dependent manner through inhibition of phosphorylation and degradation of $\mathrm{I}_{\kappa} \mathrm{B}_{2}$ and $\mathrm{I} \mathrm{KB} \beta(20)$. Aspirin may well be more potent in endothelial protection than vitamin $\mathrm{E}$ (19). 


\section{Atherosclerosis: Role of Platelets, Endothelial activation and Leukocytes}

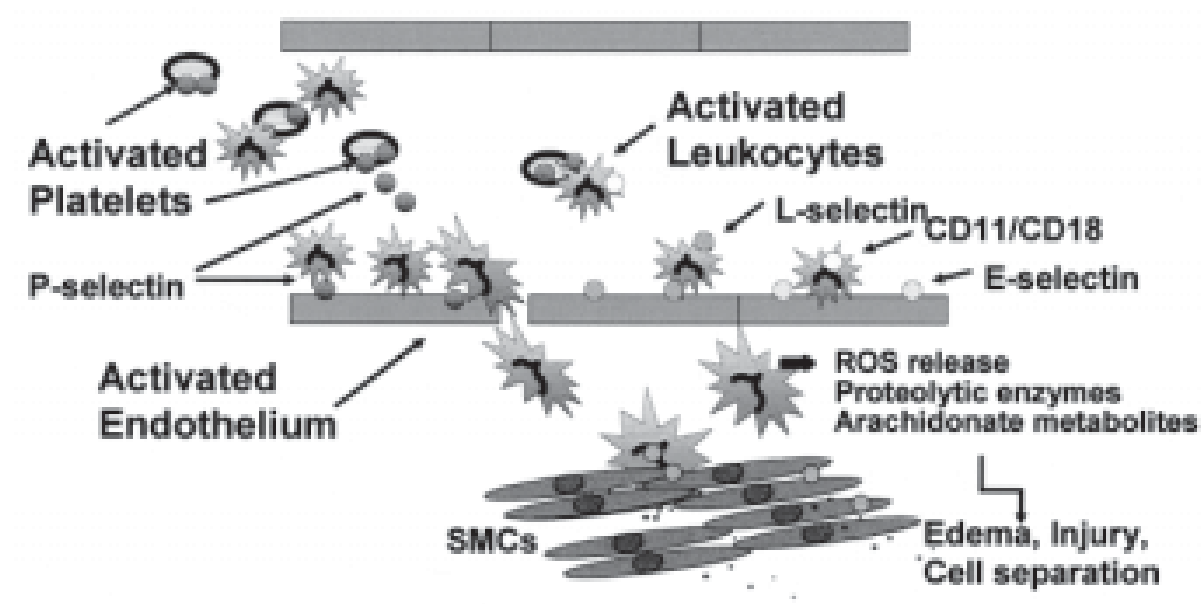

Fig. 2. Model describing early pathophysiological processes in atherosclerosis involving platelet activation, endothelial injury and dysfunction, oxidative stress and inflammation. Abbreviations: ROS-reactive oxygen species.

In recent studies, the induction of ferritin has been shown to provide marked cellular protection by rapidly sequestering free cytosolic iron, the crucial catalyst of oxygen-centered radical formation via the Fenton reaction in biological systems $(20,21)$. Cells overexpressing ferritin protein are more resistant to oxidative injury (22). Thus, ferritin, which was thought to function merely as a "housekeeper" iron storage protein, has emerged as a critical and fast-acting endogenous cytoprotectant that plays an important role in cellular antioxidant-related defense mechanisms $(23,24)$. In cultured endothelial cells, aspirin has been shown to increase the synthesis of ferritin, an effect not shown by other nonsteroidal anti-inflammatory drugs, indomethacin or diclofenac (25). Thus, it seems plausible that by increasing the synthesis of ironscavenging ferritin, aspirin may specifically withdraw iron ions from the site of oxygen radical formation, and may thus effectively interrupt the reaction cascade leading to oxidative stress and tissue damage.

The transcription factor NF-KB is a ubiquitous effector of the inflammatory response, and promotes the expression of genes encoding cytokines, cytokine receptors, chemoattractant proteins, and the adhesion molecules VCAM-1, ICAM-1, and E-selectin $(9,10)$. NF- $\kappa B$ cognate regulatory elements are present in the promoters of these three genes and are essential for their transcriptional induction by the inflammatory cytokines TNF- $\alpha$ and IL-1. Costanzo et al. (11) demonstrated that in cultured hu- man umbilical vein endothelial cells, aspirin can abolish the angiotensin II-mediated induction of NF- $\mathrm{kB}$ transcriptional activation. In contrast, exposure to indomethacin did not produce any significant inhibition of NF- $\mathrm{KB}$ activity, suggesting that these two COX inhibitors target distinct signaling pathways in this system.

\section{Effect on endothelial activation/dysfunction}

While increased platelet activity in fact plays a crucial role during acute thrombosis, it is the injured and dysfunctional endothelium that facilitates the adhesion of platelets and other blood cells to the vascular wall and can thus be considered a primary factor in atherogenesis and the formation of a thrombus (26). Pro-inflammatory cytokines released by the vessel wall components and circulating cells are considered critical to the initiation and progression of atherosclerosis. Consistent with this concept, plasma levels of macrophage colony stimulating factor (MCSF), IL-1 $\beta$, IL-6, and CRP are significantly elevated in patients with coronary atherosclerosis, in one study MCSF, IL-6, and CRP were all reduced after 6 weeks of aspirin therapy (27). This phenomenon may in part explain aspirin's therapeutic action.

As mentioned earlier, aspirin may reduce the direct toxic effect of pro-oxidative stimuli and enhance the viability of endothelial cells (18). This may be mediated by enhancement of NO synthase activity and subsequent intracellular cyclic GMP accumulation. In keeping with this 


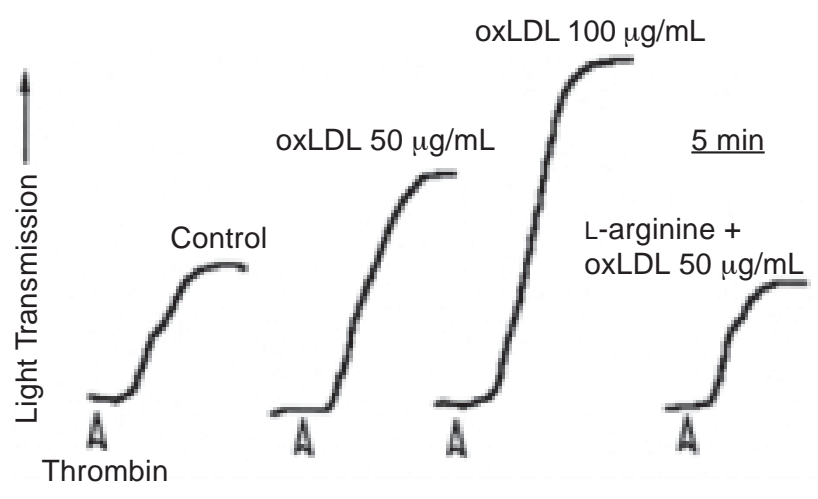

Fig. 3. Representative experiments showing stimulation of platelet aggregation when platelets were incubated with different concentrations of ox-LDL. The stimulation of platelet aggregation with 1 to $2.5 \mathrm{U} / \mathrm{mL}$ thrombin was attenuated by prior incubation of platelets with L-arginine

concept, the impaired forearm vasodilatation in hypercholestrolemic patients in response to acetylcholine was partially corrected when patients were treated with aspirin, suggesting another therapeutic action of aspirin (28). In another study hypertensive patients, a similar effect of improvement in flow-mediated dilatation and increased cGMP production was observed after treatment with aspirin (29).

Acute myocardial ischemia is associated with endothelial activation as evident from enhanced platelet adhesiveness at the site of ruptured plaques. In our laboratory, Ranganathan et al. (30) showed that aspirin was able to enhance p53 expression in human coronary artery endothelial cells exposed to ox-LDL. In addition to platelet inhibition, protection and preservation of the endothelial activity be an important target for the tissue-protective effects of aspirin.

\section{Effect on leukocyte adhesion and chemotaxis}

Increased white blood cell adhesion to the activated endothelium leads to leukocyte accumulation and transmigration to the subendothelial layers, which contribute to the progression of atherosclerotic plaques. Leukocyte adhesion is characterized by the expression of a number of molecules such as E-selectin, ICAM-1 and VCAM-1, which support their adhesion to the endothelial cells via interaction with counterligands VLA-4 (CD29/49d), LFA1 (CD11a/CD18), and Mac-1 (CD11b/CD18), all of which are expressed in human atherosclerotic plaques in large numbers $(31,32)$.

Voisard et al. (33) have shown that aspirin decreases the adherence of monocytes or CD4 (+) lymphocytes to human coronary artery endothelial cells. These investigators also showed that aspirin inhibits the proliferation of cultured human coronary artery smooth muscle cells. Weber et al. (34) also showed that aspirin in a dose-dependent fashion inhibits TNF- $\alpha$ induced NF-KB mobilization, VCAM-1 and E surface expression. Adhesion of U937 monocytes to TNF- $\alpha$-stimulated human venous endothelial cells was markedly reduced by aspirin. These effects of aspirin were not related to the inhibition of COX activity.

\section{Effect on cell proliferation}

Reactive coronary artery smooth muscle cell proliferation is a key event in the development of atherosclerosis and restenosis. As mentioned above, treatment with aspirin caused a significant decrease in the reactive proliferative response of human coronary media smooth muscle cells after the leukocyte attack. In a study in dogs fed a cholesterol-enriched diet for 8 months, aspirin treatment attenuated platelet function, prolonged bleeding time, and prevented abrupt thrombotic occlusion, but, importantly, inhibited neointimal proliferation (35).

Aspirin and sodium salicylate similarly inhibit vascular smooth muscle cell proliferation by arresting the cell cycle in the $\mathrm{G}_{1}-\mathrm{S}$ phase (36). Work from our laboratory has showed that aspirin decreases DNA and protein synthesis in endothelial cells by upregulating P53 expression (30). Bellosillo et al. (37) also showed that aspirin and salicylate induce apoptosis of chronic lymphocytic leukemia cells through the activation of caspase- 3 by COXindependent mechanisms.

These observations indicate that aspirin can decrease endothelial and smooth muscle cell proliferation through cell cycle arrest and other mechanisms. These mechanisms may contribute to the overall effects of aspirin on atherosclerosis-related diseases.

\section{Salicylate-specific actions of aspirin}

After the oral administration of an analgesic dose of aspirin, $50 \%$ is de-acetylated to salicylate immediately after absorption. The plasma half-life of aspirin is about 15 minutes whereas that of salicylate is between 2 and 30 hours depending upon the dose. Since aspirin is rapidly de-acetylated into salicylate, it is postulated that the anti-inflammatory effects of aspirin are due to the salicylate moiety.

Sodium Salicylate, unlike aspirin, is almost inactive as a direct inhibitor of COX-1 in the vascular wall (38) and does not prevent the formation of $\mathrm{TxA}_{2}$ in platelet-rich plasma. Instead, it is only a weak inhibitor of $\mathrm{TxA}_{2}$ in clotting whole blood (39). It is plausible that the anti-inflammatory effects of sodium salicylate are not dependent on the inhibition of prostaglandin synthesis.

High concentrations of salicylates have been shown to inhibit kinases including the MAPK cascade, with the 
exception of a few reports (40). NF- $\kappa B$ is regarded as a key element in the response of cells to inflammatory stimuli. Inhibition of the NF-KB pathway by aspirin and salicylate was first demonstrated by Kopp and Ghosh (41) followed by several others. Sodium salicylate also serves as a chemical trap for hydroxyl radicals, and has been shown to ameliorate hypoxia/reoxygenation injury in several tissues (42). It has also been suggested that the uncoupling of oxidative phosphorylation by salicylates decreases intra-cellular ATP formation, consequently inducing the release of adenosine into the extracellular fluids in sufficient quantity to exert anti-inflammatory effects (43).

We have observed that, like aspirin, salicylate has inhibitory effects on ox-LDL-mediated LOX-1 expression, MMP-1 expression and activity, p38MAPK and superoxide anion generation in human coronary artery endothelial cells (15). These results suggest that the novel effects of aspirin mediated by its salicylate moiety complement the role of the acetyl moiety in terms of its platelet inhibitory effect.

\section{References}

(1) Vane JR, Flower RJ, and Botting RM: History of aspirin and its mechanism of action. Stroke, IV12IV23, 1990

( 2 ) Antithrombotic Trialists' Collaboration: Collaborative meta-analysis of randomized trials of antiplatelet therapy for prevention of death, myocardial infarction, and stroke in high risk patients. BMJ, 324: 7186, 2002

(3) Antman EM, Anbe DT, Armstrong PW, Bates ER, Green LA, Hand M, Hochman JS, Krumholz HM, Kushner FG, Lamas GA, Mullany CJ, Ornato JP, Pearle DL, Sloan MA, Smith SC Jr, American College of Cardiology; American Heart Association; Canadian Cardiovascular Society: ACC/AHA guidelines for the management of patients with ST-elevation myocardial infarction-executive summary. A report of the American College of Cardiology/ American Heart Association Task Force on Practice Guidelines (Writing Committee to revise the 1999 guidelines for the management of patients with acute myocardial infarction). J Am Coll Cardiol, 44: 671-719, 2004

(4) Schror K: Aspirin and platelets: the antiplatelet action of aspirin and its role in thrombosis treatment and prophylaxis. Semin Thromb Hemost, 23: 349356, 1997

( 5 ) DeWitt DL and Smith WL: Primary structure of prostaglandin $\mathrm{G} / \mathrm{H}$ synthase from sheep vesicular gland determined from the complementary DNA sequence. Proc Natl Acad Sci USA, 85: 1412-1416, 1988
(6) Mehta JL and Nichols WW: The potential role of thromboxane inhibitors in preventing myocardial ischemic injury? Drugs, 40: 657-665, 1990

( 7 ) Chen J and Mehta JL: Role of oxidative stress in coronary heart disease. Indian Heart J, 56: 163-173, 2004

( 8 ) Ridker PM, Brown NJ, Harrison DG, Mehta JL, and Vaughn DE: Established and emerging plasma biomarkers in the prediction of first atherothrombotic event. Circulation, 109: IV6-IV19, 2004

(9) Baldwin AS Jr: The transcription factor NF-kappaB and human disease. J Clin Invest, 1: 3-6, 2001

(10) Nichols TC, Fischer TH, Deliargyris EN, and Baldwin AS Jr: Role of nuclear factor-kappaB (NF-kappa B) in inflammation, periodontitis, and atherogenesis. Ann Periodontol, 1: 20-29, 2001

(11) Costanzo A, Moretti F, Burgio VL, Bravi C, Guido F, Levrero $M$, and Puri PL: Endothelial activation by angiotensin II through NF-kappaB and p38 pathways: Involvement of NF-kappaB-inducible kinase (NIK), free oxygen radicals, and selective inhibition by aspirin. J Cell physiol, 195: 402-410, 2003

(12) Yin MJ, Yamamoto Y, and Gaynor RB: The antiinflammatory agents aspirin and salicylate inhibit the activity of I (kappa) B kinase-beta. Nature, 6706: 77-80, 1998

(13) Ehara S, Ueda M, and Naruko T: Elevated levels of oxidized low density lipoprotein show a positive relationship with the severity of acute coronary syndromes. Circulation, 103: 1955-1960, 2001

(14) Mehta JL and Li D: Identification, regulation and function of LOX-1, a novel receptor for ox-LDL. J Am Coll Cardiol, 39: 1429-1435, 2002

(15) Mehta JL, Chen J, Yu F, and Li DY: Aspirin inhibits ox-LDL-mediated LOX-1 expression and metalloproteinase-1 in human coronary endothelial cells. Cardiovasc Res, 64: 243-249, 2004

(16) Chen LY, Mehta P, and Mehta JL: Oxidized LDL Decreases L-Arginine Uptake and Nitric Oxide Synthase Protein Expression in Human Platelets. Circulation, 93: 1740-1746, 1996

(17) O'Kane PD, Queen LR, Ji Y, Reebye V, Stratton P, Jackson G, and Ferro A: Aspirin modifies nitric oxide synthase activity in platelets: effects of acute versus chronic aspirin treatment. Cardiovasc Res, 59: 152-159, 2003

(18) Grosser $\mathrm{N}$ and Schröder H: Aspirin protects endothelial cells from oxidant damage via the nitric oxide-cGMP pathway. Arterioscler Thromb Vasc Biol, 23: 1345-1351, 2003

(19) Podhaisky HP, Abate A, Polte T, Oberle S, and Schröder H: Aspirin protects endothelial cells from oxidative stress: possible synergism with vitamin E. FEBS Lett, 417: 349-351, 1997

(20) Balla G, Jacob HS, Balla J, Rosenberg M, Nath K, 
Apple F, Eaton JW, and Vercellotti GM: Ferritin: a cytoprotective antioxidant strategem of endothelium. J Biol Chem, 267: 18148-18153, 1992

(21) Cairo G, Tacchini L, Pogliaghi G, Anzon E, Tomasi $A$, and Bernelli-Zazzera A: Induction of ferritin synthesis by oxidative stress. J Biol Chem, 270: 700703, 1995

(22) Oberle S and Schröder H: Ferritin may mediate SIN1 -induced protection against oxidative stress. $\mathrm{Ni}$ tric Oxide, 1: 308-314, 1997

(23) Juckett MB, Balla J, Balla G, Jessurun J, Jacob HS, and Vercellotti GM: Ferritin protects endothelial cells from oxidized low density lipoprotein in vitro. Am J Pathol, 147: 782-789, 1995

(24) Kim YM, Bergonia H, and Lancaster JR: Nitrogen oxide-induced autoprotection in isolated rat hepatocytes. FEBS Lett, 374: 228-232, 1995

(25) Oberle S, Polte T, Abate A, Podhaisky HP, and Schröder H: Aspirin increases ferritin synthesis in endothelial cells: a novel antioxidant pathway. Circ Res, 82: 1016-1020, 1998

(26) Conti CR and Mehta JL: Acute myocardial ischemia: Role of atherosclerosis, in situ thrombosis, platelet activation, coronary vasospasm, and altered arachidonic acid metabolism. Circulation, 75: V84-V95, 1987

(27) Ikonomidis I, Andreotti F, Economou E, Stefanadis C, Toutouzas P, and Nihoyannopoulos P: Increased pro-inflammatory cytokines in patients with chronic stable angina and their reduction by aspirin. Circulation, 100: 793-798, 1999

(28) Noon JP, Walker BR, Hand MF, and Webb DJ: Impairment of forearm vasodilatation to acetylcholine in hypercholesterolemia is reversed by aspirin. Cardiovasc Res, 38: 480-484, 1998

(29) Monobe H, Yamanari H, Nakamura K, and Ohe T: Effects of low dose aspirin on endothelial function in hypertensive patients. Clin Cardiol, 24: 207-709, 2001

(30) Ranganathan S, Joseph J, and Mehta JL: Aspirin inhibits human coronary artery endothelial cell proliferation by upregulation of p53. Biochem Biophys Res Commun, 301: 143-146, 2003

(31) Cybulsky MI and Gimbrone MA: Endothelial expression of a mononuclear leukocyte adhesion molecule during atherogenesis. Science, 251: 788-791, 1991

(32) Libby P and Hansson G: Involvement of the immune system in human atherogenesis: current knowledge and unanswered questions. Lab Invest, 64: 5-15, 1991

(33) Voisard R, Fischer R, and Osswald M: Aspirin in- hibits leukocyte attack and triggered reactive cell proliferation in a 3D human coronary in vitro model. Circulation, 103: 1688-1694, 2001

(34) Weber C, Erl W, Pietsch A, and Weber PC: Aspirin inhibits nuclear factor-kappa B mobilization and monocyte adhesion in stimulated human endothelial cells. Circulation, 91: 1914-1917, 1995

(35) Anderson HV, McNatt J, Clubb FJ, Herman M, Maffrand J, DeClerck F, Ahn C, Buja LM, and Willerson JT: Platelet Inhibition Reduces Cyclic Flow Variations and Neointimal Proliferation in Normal and Hypercholesterolemic-Atherosclerotic Canine Coronary Arteries. Circulation, 104: 2331-2337, 2001

(36) Mara DE, Simoncini T, and Liao JK: Inhibition of vascular smooth muscle cell proliferation by sodium salicylate mediated by upregulation of $\mathrm{p} 21^{\text {Waf1 }}$ and p27 ${ }^{\text {Kip1 }}$. Circulation, 102: 2124-2130, 2000

(37) Bellosillo B, Pique M, Barragan M, Castano E, Villamor N, Colomer D, Montserrat E, Pons G, and Gil J: Aspirin and salicylate induce apoptosis and activation of caspases in B-cell chronic lymphocytic leukemia cells. Blood, 92:1406-1414, 1998

(38) Higgs GA, Salmon JA, Henderson B, and Vane JR: Pharmacokinetics of aspirin and salicylate in relation to inhibition of arachidonate cyclooxygenase and anti-inflammatory activity. Proceedings of the National Academy of Sciences of the United States of America, 84: 1417-1420, 1987

(39) Whittle BJ, Higgs GA, Eakins KE, Moncada S, and Vane JR: Selective inhibition of prostaglandin production in inflammatory exudates and gastric mucosa. Nature, 284: 271-273, 1980

(40) Schwenger P, Alpert D, Skolnik EY, Vilcek J: Activation of p38 mitogen-activated protein kinase by sodium salicylate leads to inhibition of tumor necrosis factor-induced Ikappa B alpha phosphorylation and degradation. Mol Cell Biol, 18: 78-84, 1998

(41) Koppe E and Ghosh S: Inhibition of NF-kappa B by sodium salicylate and aspirin. Science, 265: 956959, 1994

(42) Colantoni A, de Maria N, Caraceni P, Bernardi M, Floyd RA, and Van Thiel DH: Prevention of reoxygenation injury by sodium salicylate in isolated-perfused rat liver. Free Radic Biol Med, 25: 87-94, 1998

(43) Cronstein BN, Van de Stouwe M, Druska L, Levin $\mathrm{RI}$, and Weissmann G: Nonsteroidal anti-inflammatory agents inhibit stimulated neutrophil adhesion to endothelium: adenosine dependent and independent mechanisms. Inflammation, 18: 323-335, 1994 